\title{
Performance evaluation of two degree of freedom conventional controller adopting the smith principle for first order process with dead time
}

\author{
Belinda Sharon Bright ${ }^{1}$, R. Swarnalatha ${ }^{2}$ \\ ${ }^{1}$ Department of Electronics and Instrumentation Engg, Birla Institute of Technology and Science, \\ Pilani Dubai Campus, Academic City, Dubai, United Arab Emirates \\ ${ }^{2}$ Department of Electrical and Electronics Engg, Birla Institute of Technology and Science, \\ Pilani Dubai Campus, Academic City, Dubai, United Arab Emirates
}

\section{Article Info \\ Article history: \\ Received Aug 23, 2018 \\ Revised Mar 11, 2019 \\ Accepted Apr 4, 2019}

\section{Keywords:}

Control system

Dead time

Robust analysis

Smith predictor

Two degree of freedom

\begin{abstract}
The Proportional Integral Derivative Controller is a typical controller implemented frequently in many services and integrating the Smith predictor is an extremely useful control system structure for processes with dead time. This paper has evaluated two control schemes with the modified structures of the Smith predictor incorporating dead time compensators and conventional controllers for first order process with dead time. The disturbance response and the set point response for both the control schemes were decoupled from each other. Therefore two degrees of freedom control design was formulated, and hence the responses could be designed separately. The two control schemes have mainly two variables to be adjusted that decide the robustness and closed-loop behaviour. This paper also contains the calculation of various parameters that were used in each scheme. A comparison of the two control schemes along with the general Smith predictor control scheme was made using Simulink/Matlab. The conclusion is the second control scheme gave better response overall for the processes with dead time having dead time uncertainty and for the processes with dead time without dead time uncertainty.
\end{abstract}

Copyright (C) 2019 Institute of Advanced Engineering and Science. All rights reserved.

Corresponding Author:

Belinda Sharon Bright,

Bachelor of Electronics and Instrumentation Engineering,

Birla Institute of Technology \& Science, Pilani, Dubai Campus,

Dubai International Academic City, Dubai, U.A.E.

Email: f20140049@dubai.bits-pilani.ac.in

\section{INTRODUCTION}

Dead time between output and input is a usual occurrence in plant process systems. The existence of delay in control systems produces complication in both controller design and process study [1]. Which means, it causes the controller to overreact to a set-point or any disturbances. Therefore, to nullify the effect of dead time, Smith predictor is used which helps control the manipulated variable in the feedback loop rather than the actual process variable.

The Smith predictor (created by O.J.M. Smith in 1957) is a sort of controller which predicts what can occur in the future for control systems with time delay. The Smith principle is the prime benefit of the Smith predictor; the dead time is completely taken off from the characteristic polynomial of the control system with a feedback loop. Therefore, the outcome is a feedback loop without delay [2].

Certain modifications to the Smith predictor are required depending on the type of process. The authors in [3] have modified Smith Predictor for controlling Integrator/Time Delay processes. To achieve faster and improved response, the authors in [4] proposed alternative Smith predictor for 
controlling a process with an integrator and long dead-time. Another author in [5] presented an enhanced control of integrating cascade processes with time delay using modified Smith predictor which provides great disturbance rejection in the inner and outer loop by implementing a PID with lag filter and PD with a lead-lag filter. Also enhanced Smith predictor was proposed in [6] for networked control systems.

a. The problem

A lot of modified Smith predictors have been proposed for various types of process to provide improved results and faster response. The different types of processes range from first order to higher orders having a varying number of degrees of freedom. However, in real life, the process model may vary slightly from the actual process, and there could be a potential occurrence of dead time uncertainty [7]. However, most of the work done did not consider the effect of dead time uncertainty on their respective modified Smith predictor control structures for first order process with dead time having two degree of freedom (dof) control scheme. Therefore, the response of the modified control schemes due to the dead time uncertainty was not tested and their consequent responses was not determined.

b. The proposed solution

This paper compares the general Smith predictor scheme along with other two modified structures of Smith predictor control schemes to obtain a response with and without the effect of dead time uncertainty for two degree of freedom first order processes with dead time (FOPDT) using Matlab/Simulink software. The results of the simulation show how all the three schemes respond with and without dead time uncertainty and at the same time-solving issues of stability, slower response and large overshoot outputs.

\section{RESEARCH METHOD}

First, the General Smith predictor control scheme is described. Then, two control schemes with modified Smith predictor for first order process with dead time [8] are explained. These control schemes use PID/PI (proportional integral derivative/ proportional integral) Controller [9, 10] as it is feasible and easy to implement. All the schemes are simulated using Simulink/Matlab software. Robust stability analysis is also carried out to obtain certain parameters [11, 12]. Also, formulas derived by Morari and Zafiriou were used to calculate other parameters [13]. The remaining parameters were calculated through the trial and error method.

\subsection{General smith predictor control scheme: Two DOF controller for FOPDT}

The Smith predictor control scheme is shown in Figure 1, which has G(s) which is the first order open loop process with the delay element, the process model and a proportional integral derivative controller $[14,15]$.

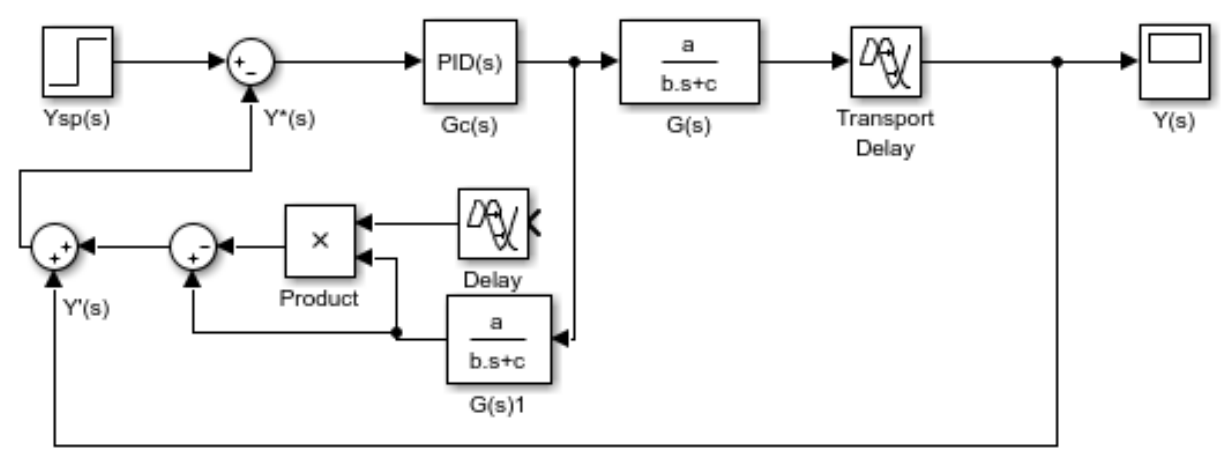

Figure 1. General Smith predictor two-DOF controller for FOPDT

As observed, there are two closed loops in the control scheme. The outside control system loop sends the end information to the input as always. However, the outer control loop does not give tolerable data as the message sent is past due to the delay that exists in the loop [16]. As a result, the plant is driven by the inner loop that has the incorrect current output data for the few seconds delay existing in the system. The Open-loop response for Figure 1 is:

$$
Y(s)=G(s) G c(s) e^{-t_{d} s} Y s p(s)
$$


To eliminate dead time information, only current information in open-loop feedback is needed, that is

$$
\begin{aligned}
& Y^{*}(s)=G c(s) G(s) Y s p(s) \\
& \text { Now, } Y^{\prime}(s)=\left\{\left(1-e^{-t_{d} s}\right) G(s)\right\} G c(s) Y s p(s)
\end{aligned}
$$

So, when $Y^{\prime}(s)$ is added with $\mathrm{Y}(\mathrm{s})$, the information to the controller is not the delayed response but the current output of the system.

$$
\begin{aligned}
Y^{*}(s) & =Y^{\prime}(s)+Y(s) \\
& =e^{-t_{d} s} G(s) G c(s) Y s p(s)+\left\{\left(1-e^{-t_{d} s}\right) G(s)\right\} G c(s) Y s p(s) \\
& =G c(s) G(s) Y s p(s)
\end{aligned}
$$

Therefore, if in the control scheme, the actual process matches the control scheme plant model perfectly, then feedback loop does not contain the dead time element $[17,18]$ and the controller can take the proper controller action.

\subsection{First control scheme: Two DOF controller for FOPDT}

The control scheme proposed by authors in [19] is shown in Figure 2. It can be noticed that the scheme is quite like that of the Smith Predictor with two additional filters. FOPDT control scheme consists of $\mathrm{F}(\mathrm{s})$ which is the $1^{\text {st }}$ DOF pre-filter, $\mathrm{C}(\mathrm{s})$ the controller, $\mathrm{Q}(\mathrm{s})$ which is the second-degree filter low-pass. It also has $\mathrm{P}(\mathrm{s})$ which is the stable process and the time delay. The feedback controller is fundamentally a PID controller.

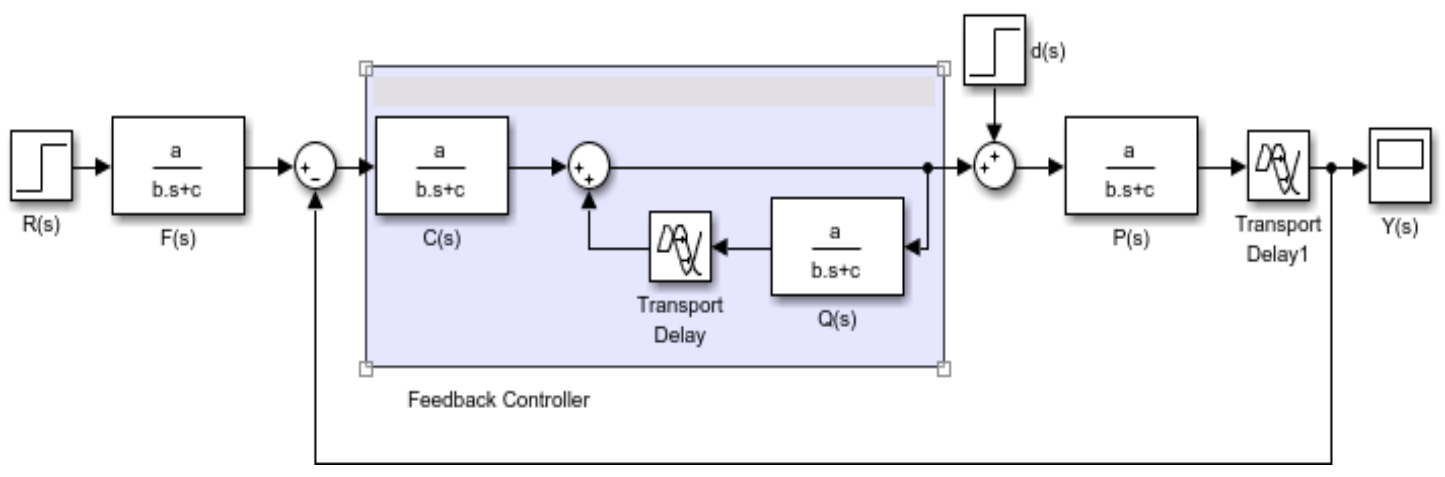

Figure 2. First control scheme Two-DOF controller for FOPDT

In the scheme, there is negative unity feedback which surrounds the positive feedback loop containing $\mathrm{Q}(\mathrm{s})$ and the time delay. Here it can be noticed that set-point response $\left[G_{r}(s)\right]$ is $e^{-\tau s} F(s) Q(s)=F(s) C(s) e^{-\tau s} P(s)$ if Controller $[C(s)]$ is designed as $\frac{Q(s)}{P(s)}$, doesn't have the $2^{\text {nd }}$ degree of freedom(dof) $\mathrm{Q}(\mathrm{s})$ in its final formula. Similarly, the disturbance response $\left[G_{d}(s)\right]$ which is $\left[1-e^{-\tau s} Q(s)\right] e^{-\tau s} P(s)$, doesn't have the $1^{\text {st }}$ degree of freedom $\mathrm{F}(\mathrm{s})$ in its final equation. Therefore, the responses that are the disturbance and the set-point respectively can be formulated individually as they are decoupled from each other. Simulink block diagram equations used for first control scheme are shown in Table 1 .

Table 1. Simulink block diagram equations for First Control Scheme

\begin{tabular}{cccc}
\hline Process without delay: & Low-Pass Filter: & Pre-Filter: & Controller: \\
\hline$P(s)=\frac{K}{T s+1}$ & $Q(s)=\frac{1}{\alpha s+1}$ & $F(s)=\frac{\alpha s+1}{\lambda s+1}$ & $C(s)=\frac{Q(s)}{P(s)}=\frac{T s+1}{K(\alpha s+1)}$ \\
\hline
\end{tabular}

In this scheme, there are two parameters to tune. That is Lambda $(\lambda)$ and Alpha $(\alpha)$. Alpha is the parameter that correlates between the robust stability and the disturbance response. Whereas, lambda is a measure of how much time is given to controller to display the output. 


\section{To calculate value of alpha}

A recommended value of $\alpha$ is (1 to 1.4) $\tau_{\Delta}$ when $\mathrm{Q}(\mathrm{s})$ is a first order filter low pass.

\section{To calculate value of lambda}

According to Morari and Zafiriou, the recommended value for $\lambda$ is:

- FOPDT: $\lambda>0.2$ open loop time constant

- SOPDT: $\lambda>0.25$ delay of the open loop process

- Ipdt: $\lambda>0.25$ delay of open loop process

\subsection{Second control scheme: Two DOF controller for FOPDT}

The control scheme proposed by authors in [20] consists of two filters. It can be noticed that its arrangement is like the Smith predictor. The arrangement consists of $F_{1}(\mathrm{~s})$ that enhances the set point response which is a traditional filter and $F_{2}(\mathrm{~s})$ that improves the disturbance rejection response which is a predictor filter. $C_{p i}(\mathrm{~s})$ is the PI Controller. $\mathrm{P}(\mathrm{s})$ is the actual process without delay. $G_{n}(\mathrm{~s})$ is a system model with the absence of the delay part and $e^{-\tau s}$ is the delay part with time delay $\tau$. The disturbance given here is $\mathrm{D}(\mathrm{s})$.

The Response of set-point as shown in Figure 3 is derived as follows:

$$
G_{r}(s)=\frac{Y(s)}{Y_{s p}(s)}=\frac{F_{1}(s) P_{n}(s) C_{p i}(\mathrm{~s})}{1+G_{n}(s) C_{p i}(\mathrm{~s})}
$$

Whereas, the disturbance response of the scheme as shown in Figure 3 is given by:

$$
Q(s)=\frac{Y(s)}{D(s)}=P_{n}(s)\left[1-\frac{C_{p i}(\mathrm{~s}) P_{n}(s) F_{2}(s)}{1+C_{p i}(\mathrm{~s}) G_{n}(s)}\right]=P_{n}(s) \lambda
$$

The above equations hold well if the actual process matches the process model.

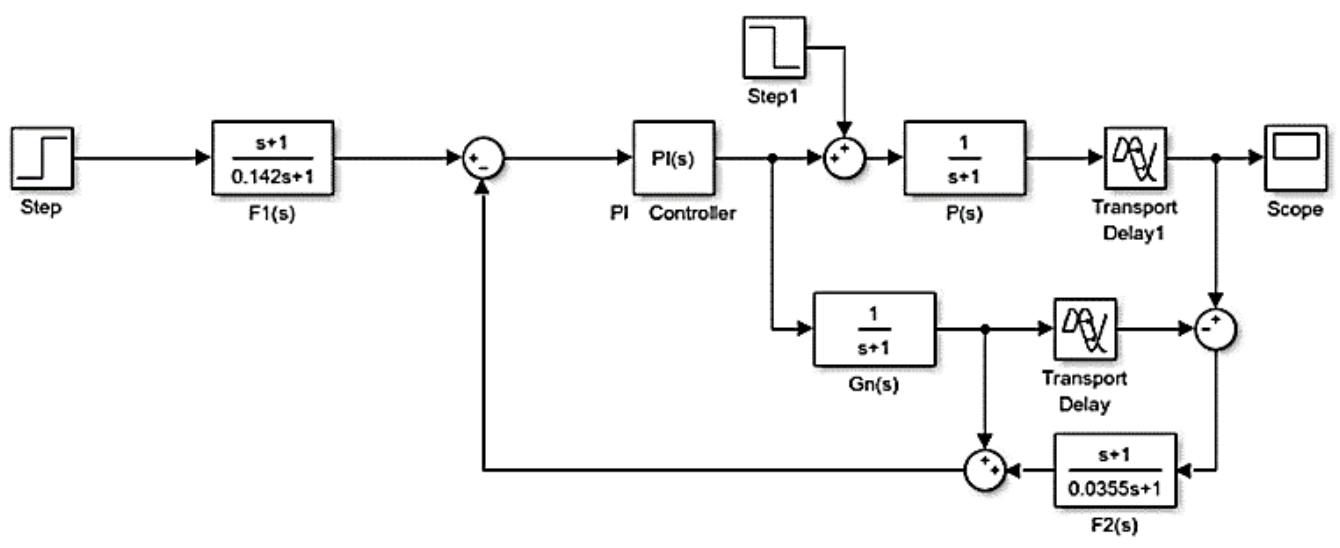

Figure 3. Second control scheme Two-DOF controller for FOPDT

In this scheme, there are two parameters to tune. That is, $K_{0}$ and $K_{1} . K_{1}$ is the parameter that correlates between the robust stability and the disturbance response. Whereas, $K_{0}$ is set to obtain the process response to reach the desired value very fast. Table 2 and Table 3 presents the formulas utilized for various parameters.

Table 2. Simulink block diagram equations for Second Control Scheme

\begin{tabular}{cccc}
\hline Actual Process without delay: & Traditional Filter: & Predictor Filter: & Process model without delay: \\
\hline$P(\mathrm{~s})=\frac{K}{T s+1}$ & $F_{1}(s)=\frac{T_{0} s+1}{K_{0} s+1}$ & $F_{2}(s)=\frac{T_{0} s+1}{K_{1} s+1}$ & $G_{1}(s)=\frac{1}{T s+1}$ \\
\hline
\end{tabular}

\begin{tabular}{|c|c|c|c|c|}
\hline$T_{0}=$ & $K_{0}=$ & $K_{1}=$ & PI tuning parameters: & $\lambda=$ \\
\hline $\begin{array}{l}T \text { (open loop time } \\
\text { constant of the }\end{array}$ & $\frac{T_{0}}{7}=\frac{T}{7}$ (determined by the & $\begin{array}{c}=\frac{T_{0}}{14} . \text { Here } K_{1} \text { is tuned by } \\
\text { trial and error method }\end{array}$ & $\mathrm{Kc}=\frac{0.5 T}{K \tau}($ proportional gain $)$ & $e^{-\tau s}$ \\
\hline system) & & which indirectly changes $\lambda$ & (integral time) & $\overline{K_{1} s+1}$ \\
\hline
\end{tabular}

Table 3. Parameters used in Simulink diagram equations for Second Control Scheme

Performance evaluation of two degree of freedom conventional controller ... (Belinda Sharon Bright) 


\section{RESULTS AND ANALYSIS}

In this section, the results of the research are explained. The analysis and comparison of general Smith predictor scheme, first modified Smith predictor scheme and second modified Smith predictor control scheme for two cases are described. The first case is for FOPDT process without dead time uncertainty [nominal case] and the second one for FOPDT process with dead time uncertainty. The FOPDT control system utilised to proceed with the comparative analysis is:

$$
\mathrm{T}(\mathrm{s})=\mathrm{P}(\mathrm{s}) e^{-\tau s}=\frac{e^{-0.5 s}}{1+s}
$$

This first order process with the dead time taken for the study has a dead time uncertainty $\left(\tau_{\Delta}\right)$ of $1 \mathrm{sec}$ and a step input $\mathrm{d}=-0.4$ that is the disturbance which acts at $5 \mathrm{secs}$. The open loop time constant is taken as 1 secs with dead time of $0.5 \mathrm{secs}$.

\subsection{Nominal case (no dead time uncertainty)}

\subsubsection{General smith predictor control scheme}

Using the calculated and considered values for the parameters as shown in Table 4, the Simulink diagram as shown in Figure 4 and Figure 5 is implemented in Matlab software. We see that for a step input with magnitude 3 in Figure 5. The dead time was found to be 0.261 secs. The rise time is 2.273 sec. The settling time at $2 \%$ tolerance is 11.9 secs. The peak time is 15.01 secs. Finally, the percentage overshoot is $\frac{(3-3)}{3} \times 100=0 \%$.

Table 4. The simulink block diagram equations for general control scheme (nominal case)

\begin{tabular}{ccc}
\hline The PI tuning parameters: & Actual Process without delay: & Process model without delay: \\
\hline - Proportional gain, $\mathrm{Kc}=\frac{0.5 T}{K \tau}=\frac{0.5 \times 1}{1 \times 0.5}=1$ & $G(\mathrm{~s})=\frac{K}{T s+1}=\frac{1}{s+1}$ & $G_{1}(s)=\frac{1}{T s+1}=\frac{1}{s+1}$ \\
- Integral gain, $\tau_{I}=T=1$ & & \\
\hline
\end{tabular}

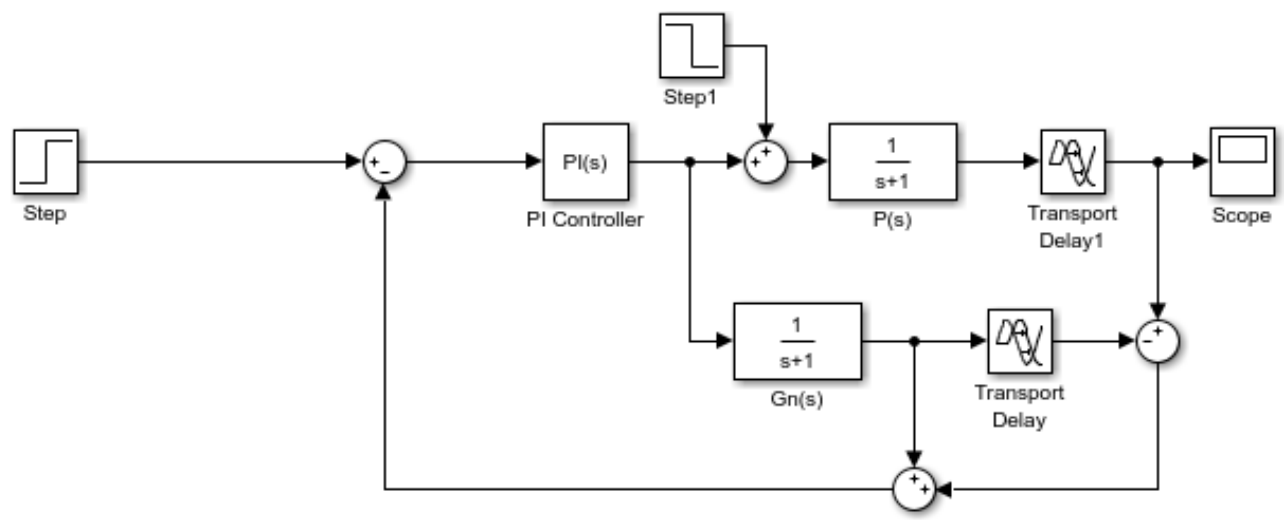

Figure 4. General control scheme alternate simulink diagram nominal case for FOPDT

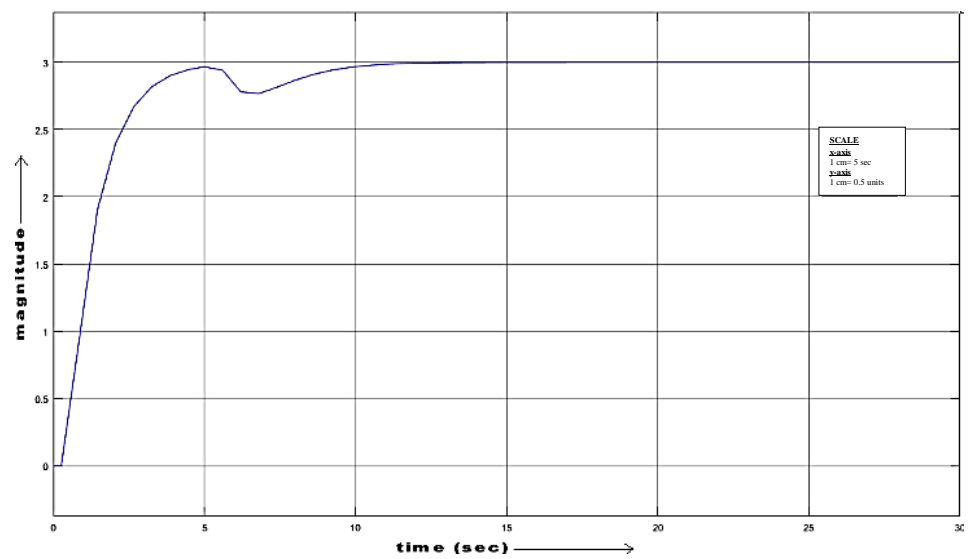

Figure 5. General control scheme response graph nominal case for FOPDT 


\subsubsection{First modified smith predictor control scheme}

To determine the value of Lambda $(\lambda)$, according to Morari and Zafiriou, the recommended value to calculate $\lambda$ for FOPDT is $\lambda>0.2$ open loop time constant. Hence, Lambda $(\lambda)=0.3 \mathrm{~s}$ was finally considered. Using all the calculated and considered values for the parameters as shown in Table 5, the Simulink diagram refer Figure 6 and Figure 7 is implemented in Matlab software.

Table 5. For different values of alpha taken randomly, their respective $\mathrm{F}(\mathrm{s}), \mathrm{C}(\mathrm{s})$ and $\mathrm{Q}(\mathrm{s})$ are calculated

\begin{tabular}{cccc}
\hline & $\alpha=0.01$ & $\alpha=0.2$ & $\alpha=0.4$ \\
\hline The 2n ${ }^{\text {nd }}$ degree low-pass filter: & $Q(s)=\frac{1}{0.01 s+1}$ & $Q(s)=\frac{1}{0.2 s+1}$ & $Q(s)=\frac{1}{0.4 s+1}$ \\
The Controller: & $C(s)=\frac{T s+1}{k(\alpha s+1)}=\frac{s+1}{(0.01 s+1)}$ & $C(s)=\frac{T s+1}{k(\alpha s+1)}=\frac{s+1}{(0.2 s+1)}$ & $C(s)=\frac{T s+1}{k(\alpha s+1)}=\frac{s+1}{(0.4 s+1)}$ \\
The 1 ${ }^{\text {st }}$ dof pre-filter: & $F(s)=\frac{\alpha s+1}{\lambda s+1}=\frac{0.01 s+1}{0.3 s+1}$ & $F(s)=\frac{\alpha s+1}{\lambda s+1}=\frac{0.2 s+1}{0.3 s+1}$ & $F(s)=\frac{\alpha s+1}{\lambda s+1}=\frac{0.4 s+1}{0.3 s+1}$ \\
\hline
\end{tabular}
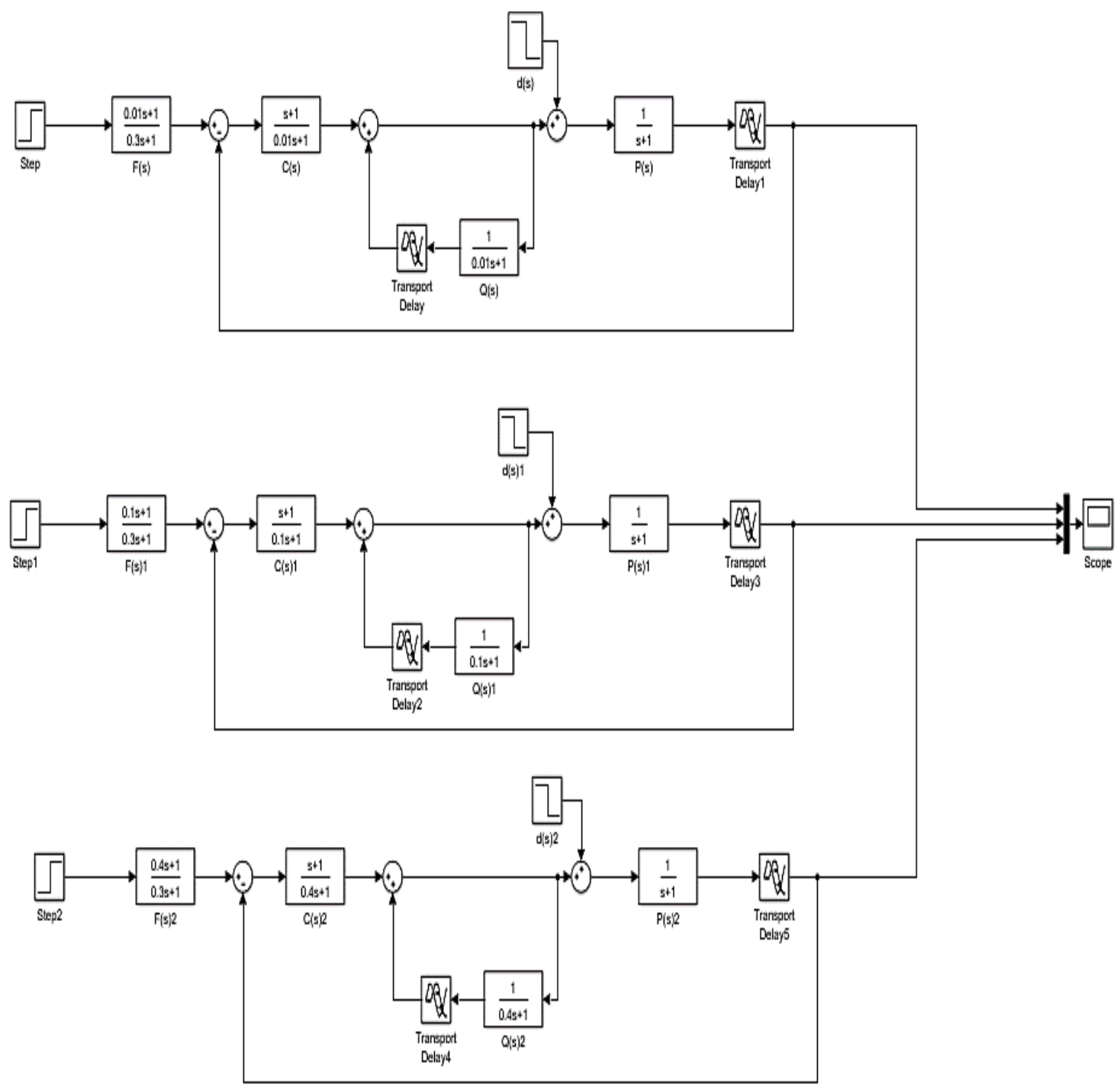

Figure 6. First control scheme simulink diagram nominal case for FOPDT 


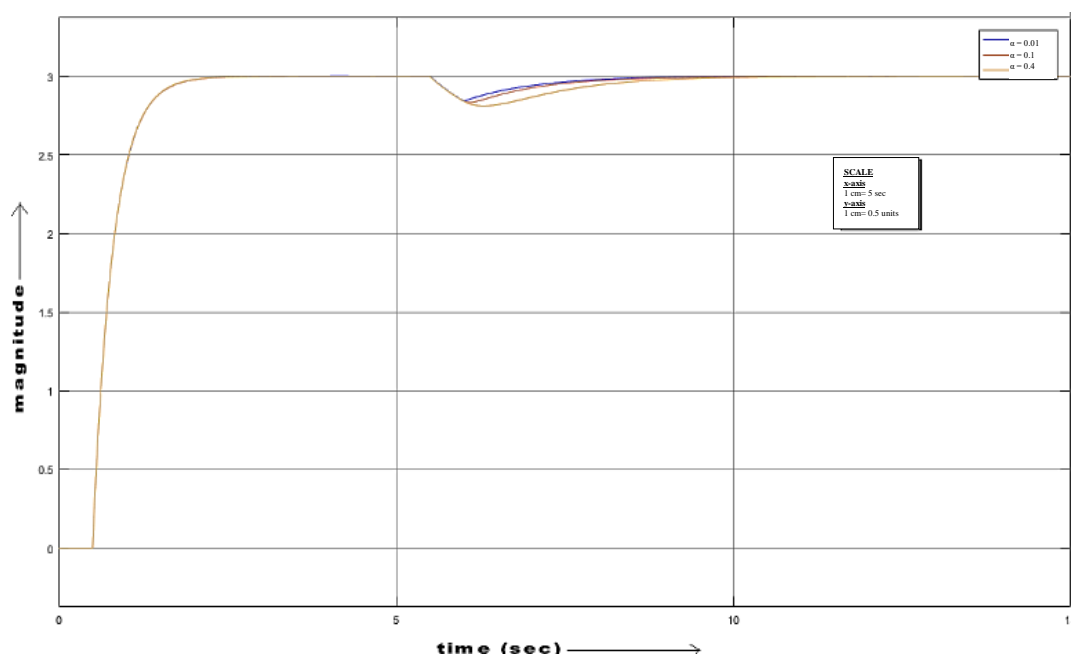

Figure 7. First control scheme response graph nominal case for FOPDT

We see that for a step input with magnitude 3 in Figure 9, using a trial and error method, $\alpha=0.01$ gives less undershoot as compared to the other two alpha values. The dead time was found to be 0.499 secs. The rise time is $647.759 \mathrm{~ms}$. The settling time at $2 \%$ tolerance is 9.4 secs and the peak time is 2.5 secs. Finally, the percentage overshoot is $\frac{(3-3)}{3} \times 100=0 \%$.

\subsubsection{Second modified smith predictor control scheme}

Using the calculated and considered values for the parameters as shown in Table 6 and Table 7, the Simulink diagram as shown in Figure 8 and Figure 9 is implemented in Matlab software.

Table 6. Parameters used in simulink diagram equations for second control scheme (nominal case)

\begin{tabular}{|c|c|c|c|c|}
\hline$T_{0}=$ & $K_{0}=$ & $K_{1}=$ & PI tuning parameters: & $\lambda=$ \\
\hline $\begin{array}{l}T=1 \text { (open } \\
\text { loop time } \\
\text { constant of the } \\
\text { system) }\end{array}$ & $\begin{array}{c}\frac{T_{0}}{7}=\frac{T}{7}= \\
0.142\end{array}$ & $\begin{array}{l}\frac{T_{0}}{14}=0.071 \text { (we take here } K_{1}=0.0355 \text { to } \\
\text { get better response as the value can be } \\
\text { tuned by trial and error method also.) }\end{array}$ & $\begin{array}{l}\text { Proportional gain, Kc } \\
\quad=\frac{0.5 T}{K \tau}=\frac{0.5 \times 1}{1 \times 0.5}=1 \\
\text { Integral gain, } \tau_{I}=T=1\end{array}$ & $\begin{aligned} & 1-\frac{e^{-\tau S}}{K_{1} s+1} \\
= & 1-\frac{e^{-0.5 s}}{0.0355 s+1}\end{aligned}$ \\
\hline
\end{tabular}

Table 7. The simulink block diagram equations for second control scheme (nominal case)

\begin{tabular}{cccc}
\hline $\begin{array}{c}\text { Actual Process } \\
\text { without delay: }\end{array}$ & Traditional Filter: & Predictor Filter: & Process model without delay: \\
\hline$P(\mathrm{~s})=\frac{K}{T s+1}=\frac{1}{s+1}$ & $F_{1}(s)=\frac{T_{0} s+1}{K_{0} s+1}=\frac{s+1}{0.142 s+1}$ & $F_{2}(s)=\frac{T_{0} s+1}{K_{1} s+1}=\frac{s+1}{0.0355 s+1}$ & $G_{1}(s)=\frac{1}{T s+1}=\frac{1}{s+1}$ \\
\hline
\end{tabular}

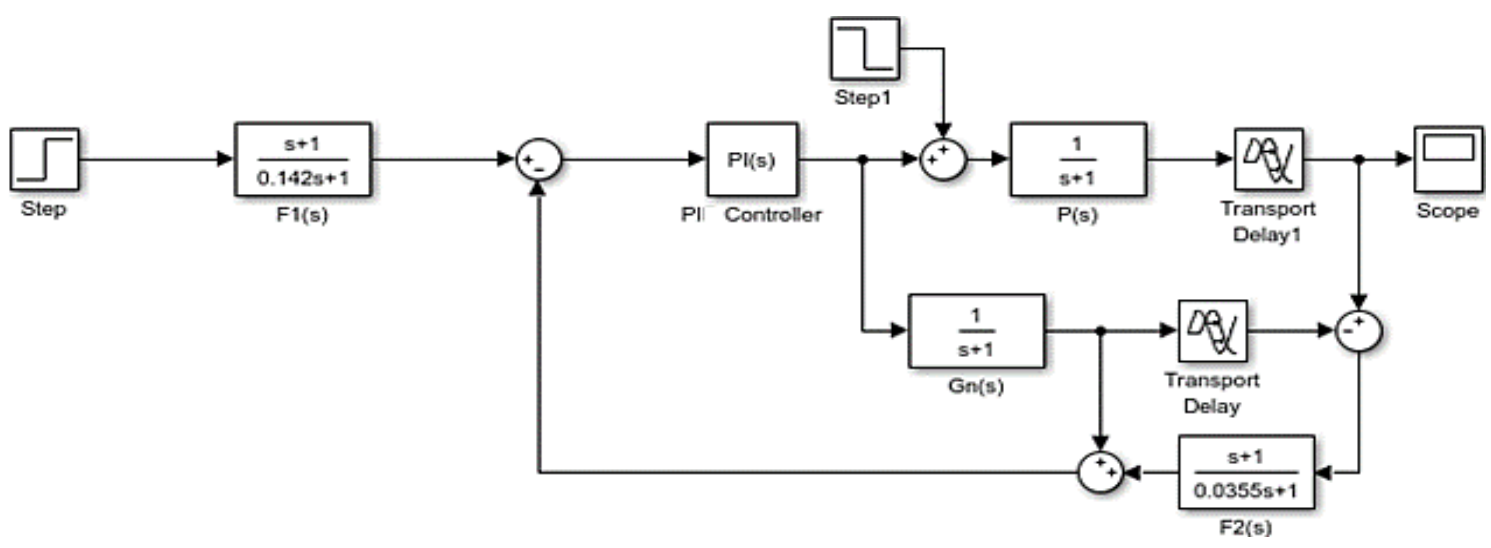

Figure 8. Second control scheme simulink diagram nominal case for FOPDT 


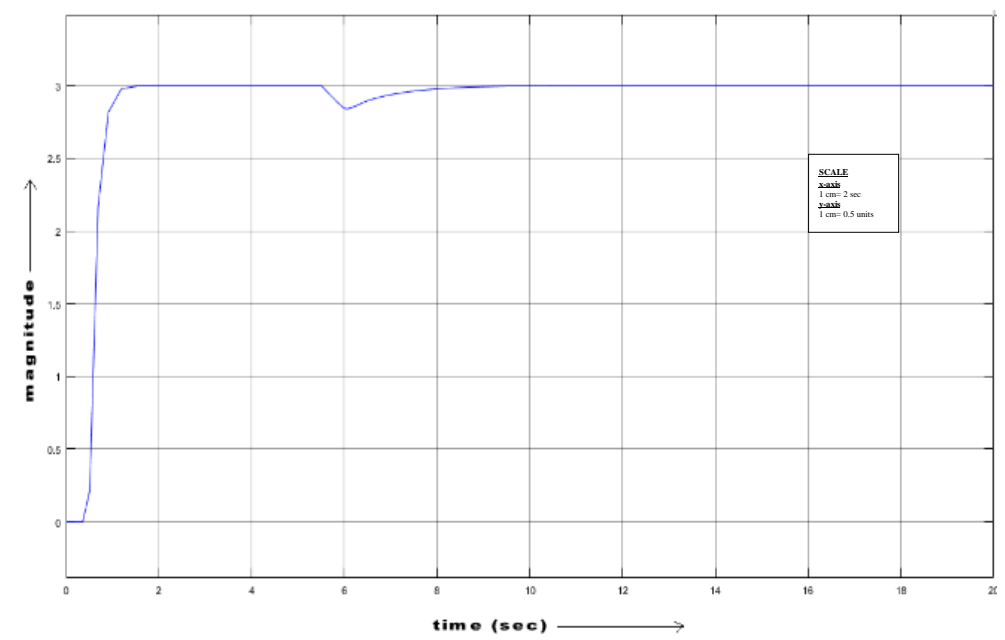

Figure 9. Second control scheme response graph nominal case for FOPDT

Therefore, for a step input of magnitude 3 in Figure 14. The dead time is 0.355 secs. The rise time is $349.277 \mathrm{~ms}$. The settling time at $2 \%$ tolerance is 7.35 secs. The peak time is 1.5 secs, and the percentage overshoot is $\frac{(3-3)}{3} \times 100=0 \%$.

\subsection{Dead time uncertainty case}

\subsubsection{General smith predictor control scheme}

Using the calculated and considered values for the parameters as shown in Table 8, the Simulink diagram as shown in Figure 10 and Figure 11 is implemented in Matlab software.

Table 8 . The simulink block diagram equations for general control scheme (dead time uncertainty case)

\begin{tabular}{ccc}
\hline The PI tuning parameters: & Actual Process without delay & Process model without delay: \\
\hline Proportional gain, $\mathrm{Kc}=\frac{0.5 T}{K \tau}=$ & $P(\mathrm{~s})=\frac{K}{T s+1}=\frac{1}{1+s}$ & $G_{1}(s)=\frac{1}{T s+1}=\frac{1}{s+1}$ \\
$\frac{0.5 \times 1}{1 \times 0.5}=1$ & & \\
Integral gain, $\tau_{I}=T=1$ &
\end{tabular}

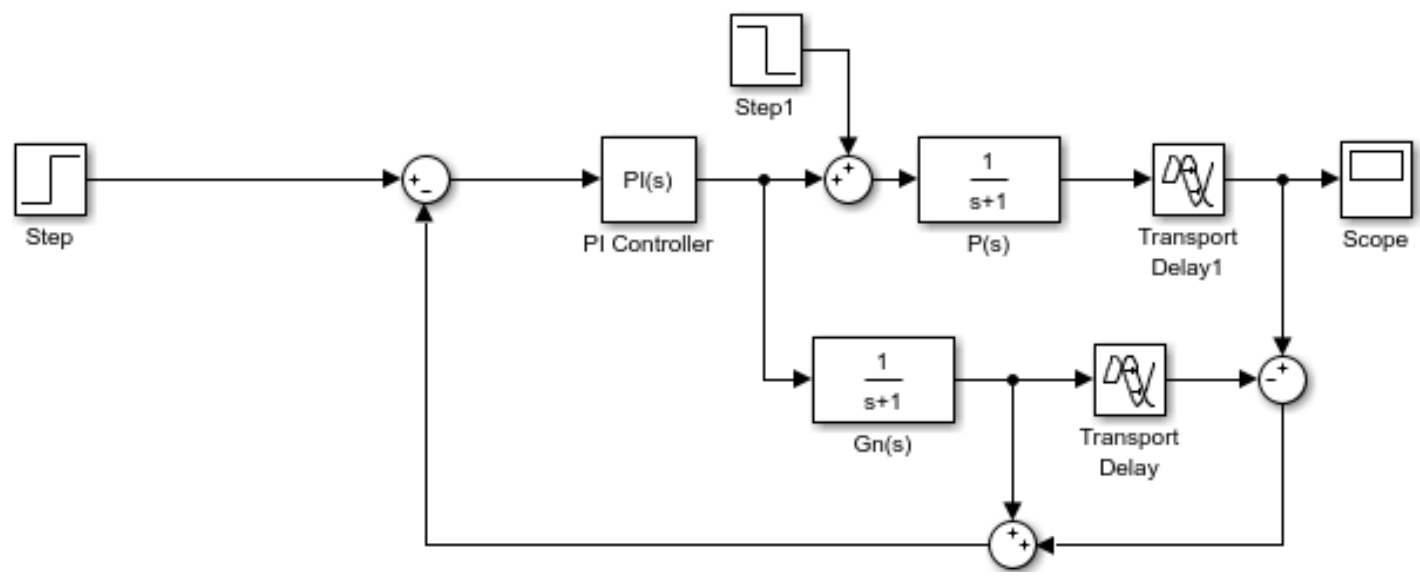

Figure 10. General control scheme alternate simulink diagram uncertainty case for FOPDT 


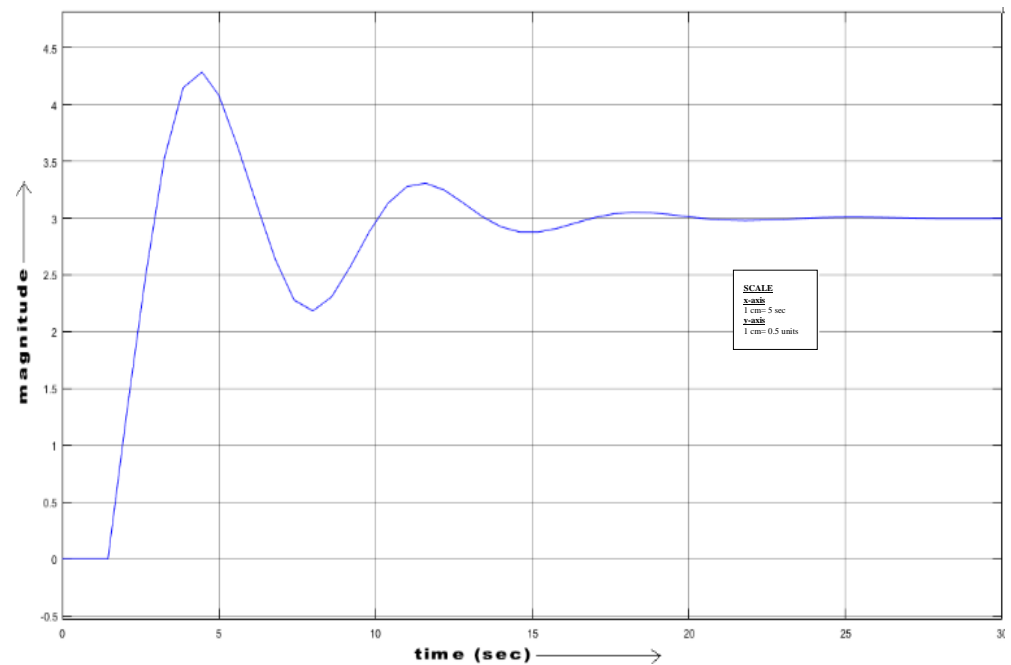

Figure 11. General control scheme response graph uncertainty case for FOPDT

We see that for a step input with magnitude 3 in Figure 7. The dead time was found to be 1.46 secs. The rise time is $1.164 \mathrm{sec}$. The settling time at $2 \%$ tolerance is 22.6 secs and the peak time is 4.462 secs. Finally, the percentage overshoot is $\frac{(4.286-3)}{3} \times 100=42.86 \%$.

\subsubsection{First modified smith predictor control scheme}

Using the robust stability analysis, different values of $\alpha$ are taken and bode plot for each is plotted to obtain a satisfactory $\alpha$ value as shown in Figure 14. Finally, $\operatorname{Alpha}(\alpha)=1.2$ was chosen to obtain better robust stability even when dead time uncertainties exist in the system as shown in Figure 12. To calculate $\lambda$, according to Morari and Zafiriou, the recommended value to calculate $\lambda$ for FOPDT is $\lambda>0.2$ open loop process time constant. Hence, Lambda $(\lambda)=0.3 \mathrm{~s}$ was finally considered. Using all the calculated and considered values for the parameters as shown in Table 9, the Simulink diagram as shown in Figure 12, Figure 13 and Figure 14 is implemented in Matlab software.

Table 9. The simulink block diagram equations for first control scheme (dead time uncertainty case)

\begin{tabular}{cccc}
\hline Process without delay: & Low-Pass Filter: & Pre-Filter: & Controller: \\
\hline$P(s)=\frac{K}{T s+1}=\frac{1}{s+1}$ & $Q(s)=\frac{1}{\alpha s+1}=\frac{1}{1.2 s+1}$ & $F(s)=\frac{\alpha s+1}{\lambda s+1}=\frac{1.2 s+1}{0.3 s+1}$ & $C(s)=\frac{Q(s)}{\mathrm{P}(s)}=\frac{T s+1}{k(\alpha s+1)}=\frac{s+1}{(1.2 s+1)}$ \\
\hline
\end{tabular}

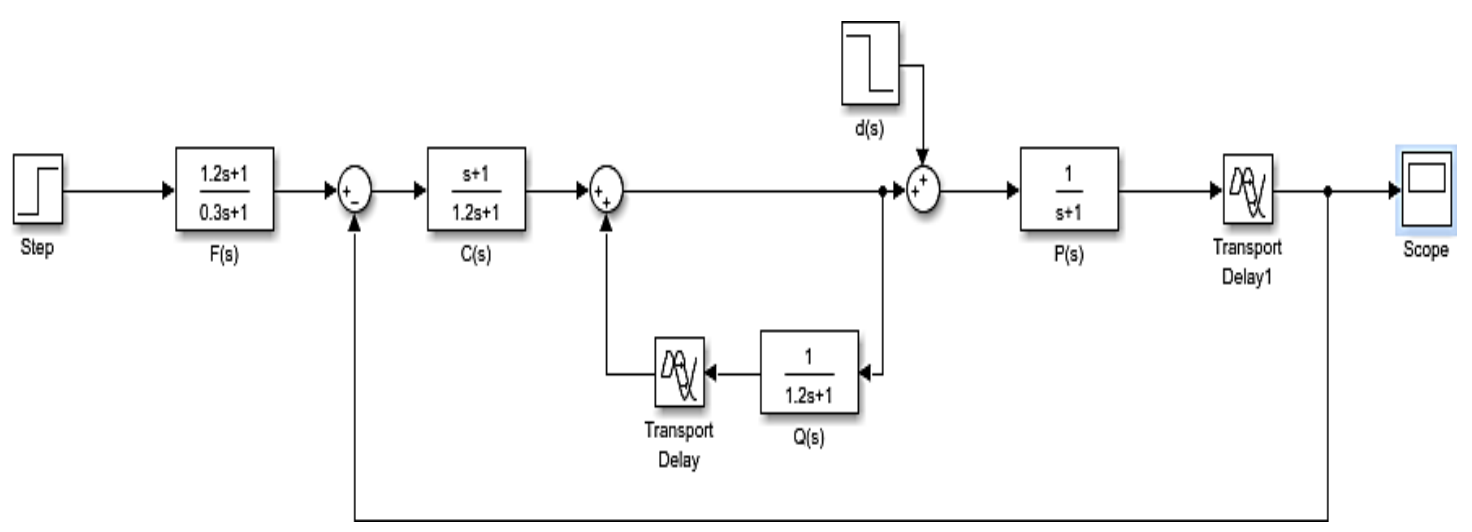

Figure 12. First control scheme: simulink diagram uncertainty case for FOPDT 


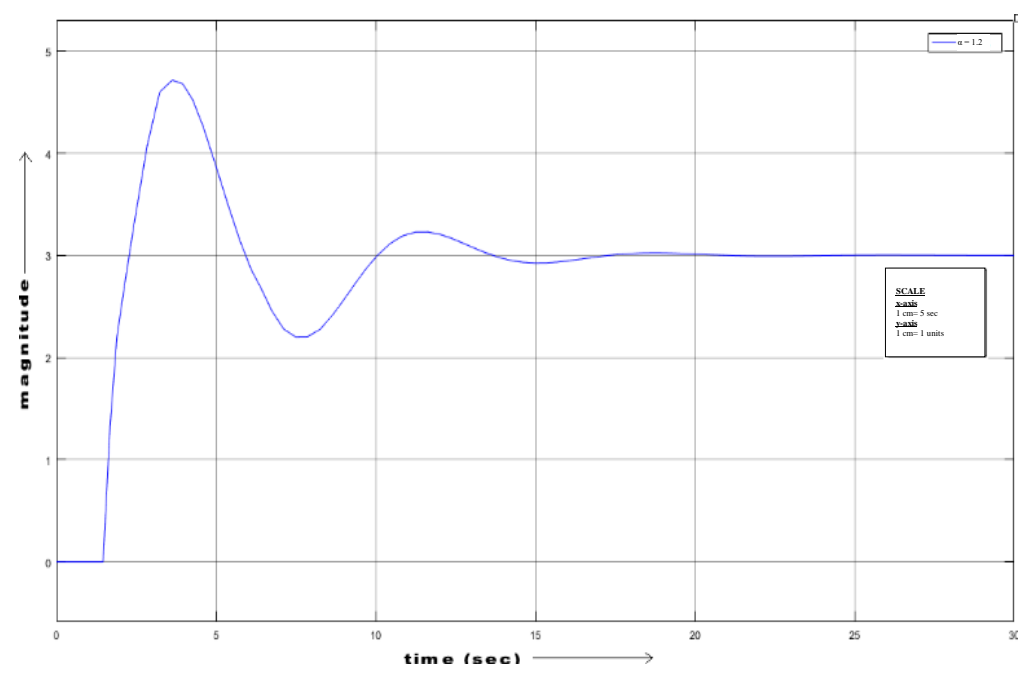

Figure 13. First control scheme response graph uncertainty case for FOPDT

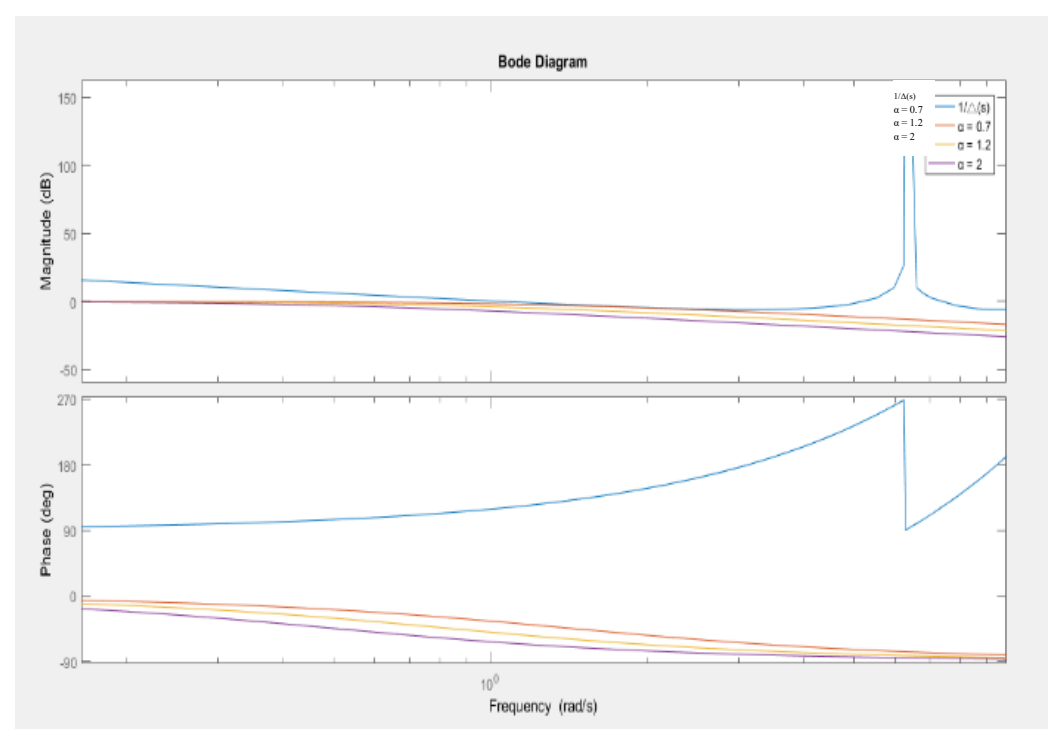

Figure 14. Stability analysis bode plot for various alpha values

We see that for a step input with magnitude 3 in Figure 12. The dead time was found to be 1.463 secs. The rise time is $613.491 \mathrm{~ms}$. The settling time at $2 \%$ tolerance is 16.5 secs and the peak time is 3.8 secs. Finally, the percentage overshoot is $\frac{(4.7-3)}{3} \times 100=56.7 \%$.

\subsubsection{Second modified smith predictor control scheme}

Using the calculated and considered values for the parameters as shown in Table 10 and Table 11, the Simulink diagram as shown in Figure 15 and Figure 16 is implemented in Matlab software.

Table 10. Parameters used in simulink for second control scheme (dead time uncertainty case)

\begin{tabular}{|c|c|c|c|c|}
\hline$T_{0}=$ & $K_{0}=$ & $K_{1}=$ & PI tuning parameters: & $\lambda=$ \\
\hline $\begin{array}{c}T=1 \text { (open loop } \\
\text { time constant of the } \\
\text { system) }\end{array}$ & $\begin{array}{c}\frac{T_{0}}{7}=\frac{T}{7}= \\
0.142\end{array}$ & $\begin{array}{l}\frac{T_{0}}{14}=0.071 \text { (we take here } K_{1}=4 \text { to } \\
\text { get better response as the value can be } \\
\text { tuned by trial and error method also.) }\end{array}$ & $\begin{array}{l}\text { Proportional gain, Kc } \\
\qquad=\frac{0.5 T}{K \tau}=\frac{0.5 \times 1}{1 \times 0.5}=1 \\
\text { Integral gain, } \tau_{I}=1\end{array}$ & $\begin{array}{l}1-\frac{e^{-\tau s}}{K_{1} s+1} \\
=1-\frac{e^{-0.5 s}}{4 s+1}\end{array}$ \\
\hline
\end{tabular}


Table 11. The simulink block diagram equations for second control scheme (dead time uncertainty case)

\begin{tabular}{cccc}
\hline Actual Process without delay: & Traditional Filter: & Predictor Filter: & Process model without delay: \\
\hline$P(\mathrm{~s})=\frac{K}{T s+1}=\frac{1}{s+1}$ & $F_{1}(s)=\frac{T_{0} s+1}{K_{0} s+1}=\frac{s+1}{0.142 s+1}$ & $F_{2}(s)=\frac{T_{0} s+1}{K_{1} s+1}=\frac{s+1}{4 s+1}$ & $G_{1}(s)=\frac{1}{T s+1}=\frac{1}{s+1}$
\end{tabular}

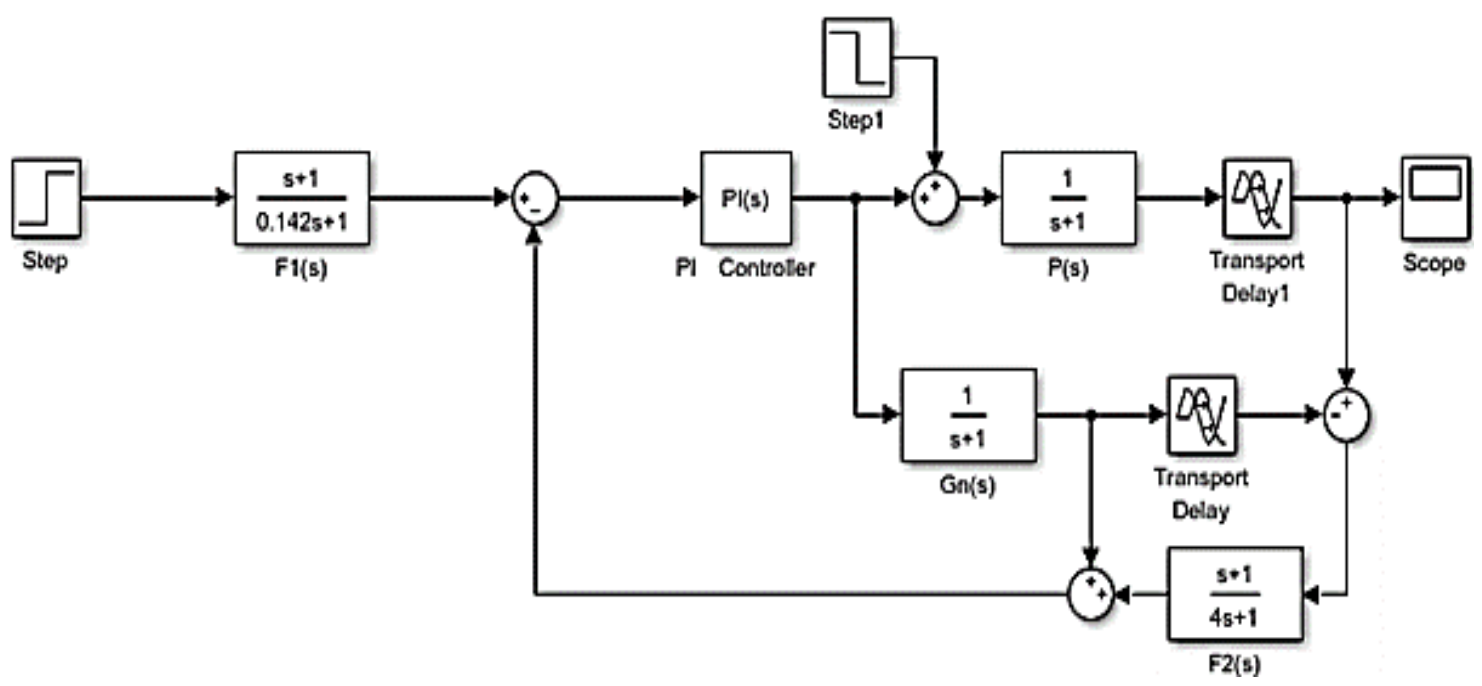

Figure 15. Second control scheme simulink diagram uncertainty case for FOPDT

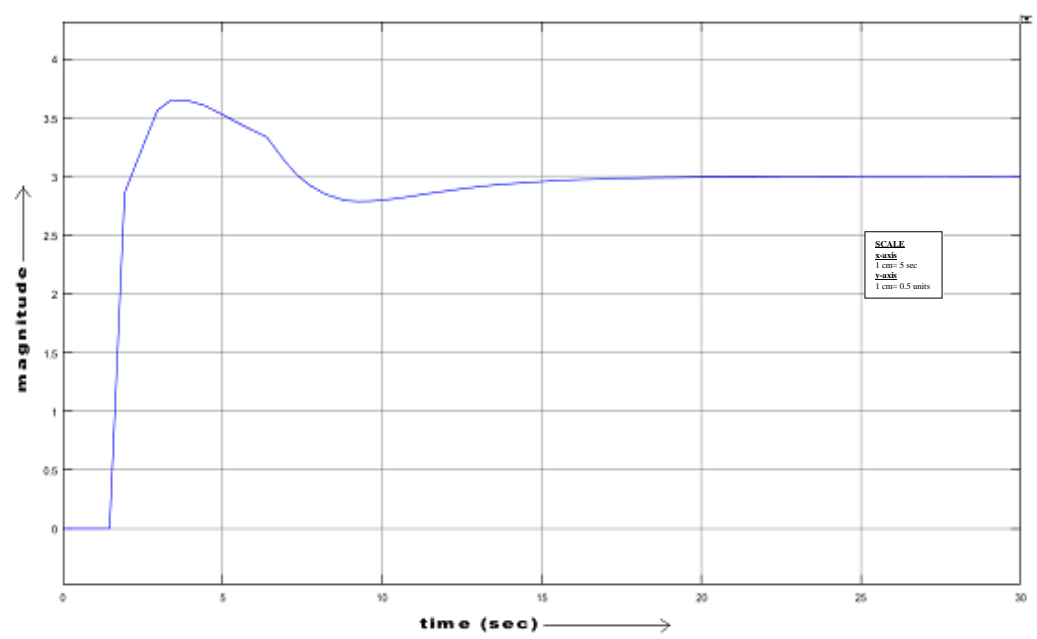

Figure 16. Second control scheme response graph uncertainty case for FOPDT

Therefore, for a step input of magnitude 3 in Figure 16. The dead time is 1.465 secs. The rise time is $393.882 \mathrm{~ms}$. The settling time at $2 \%$ tolerance is 16.6 secs. The peak time is 3.611 secs, and the percentage overshoot is $\frac{(3.649-3)}{3} \times 100=21.63 \%$.

\section{CONCLUSION}

For systems without dead time uncertainty, depending on the parameter required, either the general scheme can be used for better dead time response or the second scheme can be beneficial in terms of reduced time taken to reach preferred output. However, for a system with dead time uncertainty, it is preferable to use the second control scheme for overall better response. Importantly, the above observations hold good if the actual process matches the system model in the second scheme. 
Further research can be done by using higher order processes to check various outputs from these three schemes and decide which scheme is best suited for the tested process. Although, using processes with more degree of freedom will involve a larger number of parameter values to be utilised which can be more complex.

\section{REFERENCES}

[1] K. Wantanabe and M. Ito, "A Process-Model Control for Linear Systems with Delay," IEEE Transactions on Automatic Control, vol/issue: 6(6), pp. 1261-1268, 1981.

[2] O. J. M. Smith, "Closed control of loops with dead time," Chemical Engineering Progress, vol. 53, pp. 217-219, 1957.

[3] Y. X. Sun and W. D. Zhang, "Modified Smith Predictor for Controlling Integrator/Time Delay Processes," Ind. Eng. Chem. Res, vol. 35, pp. 2769-2772, 1996.

[4] K. J. Astrom, et al., "A new Smith predictor for controlling a process with an integrator and long dead-time," IEEE Trans. Autom. Control, vol. 39, pp. 344, 1994.

[5] S. Uma, et al., "Enhanced control of integrating cascade processes with time delay using modified Smith predictor," Chemical Engineering Science, vol/issue: 65(3), pp. 1065-1075, 2010.

[6] Y. Wu and Y. Wu, "A Novel Predictive Control Scheme with an Enhanced Smith Predictor for Networked Control System," Automatic Control and Computer Sciences, vol/issue: 52(2), pp. 126, 2018.

[7] J. Moser, et al., "Uncertainty of dead time estimation in ICP-MS," Journal of Analytical Atomic Spectrometry, vol/issue: 18(5), pp. 508-511, 2003.

[8] T. Hagglund, "An industrial dead-time compensating PI controller," Control Engineering Practice, vol/issue: 4(6), pp. 749-756, 1996

[9] A. Uthman and S. Sudin, "Antenna Azimuth Position Control System using PID Controller \& State-Feedback Controller Approach," International Journal of Electrical and Computer Engineering (IJECE), vol/issue: 8(3), pp. 1539-1550, 2018.

[10] O. Ibrahim, et al., "Performance Evaluation of Three PID Controller Tuning Algorithm on a Process Plant," International Journal of Electrical and Computer Engineering (IJECE), vol/issue: 5(5), pp. 1075-1082, 2015.

[11] C. B. S. Dutra, et al., "Robustness Analysis of DMC for First Order plus Dead-time Processes," IFAC Proceedings Volume, vol/issue: 35(1), pp. 367-372, 2002.

[12] V. Vijayan, et al., "Stability Analysis of First Order Plus Time Delay System under PI \& PID Control for Simultaneous Parameter Variation," 2005 Annual IEEE India Conference - Indicon, pp. 73-77, 2005.

[13] M. Morari and E. Zafiriou, "Robust Process Control," Prentice Hall, 1989.

[14] N. J. S. Amlashi, "Design and Implementation of Fuzzy Position Control System for Tracking Applications and Performance Comparison with Conventional PID," IAES International Journal of Artificial Intelligence, vol/issue: 1(1), pp. 31-44, 2012.

[15] M. Shamsuzzoha, "IMC based robust PID controller tuning for disturbance rejection," Journal of Central South University, vol/issue: 23(3), pp. 581-597, 2016.

[16] P. Albertos and P. Garcia, "Robust control design for long time-delay systems," Journal of Process Control, vol/issue: 19(10), pp. 1640-1648, 2009.

[17] D. Gu, et al., "Analytical design of two-degree- of freedom control scheme for open-loop unstable processes with time delay," Journal of Process Control, vol. 15, pp. 559-572, 2005.

[18] N. Abe and K. Yamanaka, "Smith predictor control and internal model control-a tutorial," SICE Annual Conference in Fukui, vol. 1, pp. 1383-1387, 2003.

[19] H. X. Li and Q. C. Zhong, "2-Degree-of-Freedom Proportional-Integral-Derivative-Type Controller Incorporating the Smith Principle for Processes with Dead Time,” Ind. Eng. Chem. Res, vol. 41, pp. 2448-2454, 2002.

[20] K. Kirtania and M. A. A. S. Choudhury, "A Two-Degree- of-Freedom Dead Time Compensator for Stable Processes with Dead Time," Proceedings of the 2011 4th International Symposium on Advanced Control of Industrial Processes, pp. 385-390, 2011.

\section{BIOGRAPHIES OF AUTHORS}

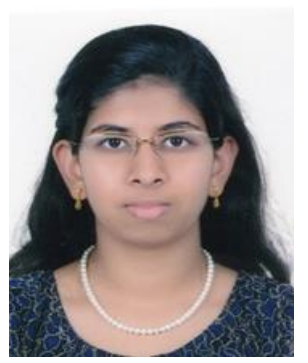

Belinda Sharon Bright is currently pursuing Bachelor of Engineering with Honors in Electronics and Instrumentation Engineering in Bits Pilani Dubai Campus. Her area of interest is Process Control \& Instrumentation. 


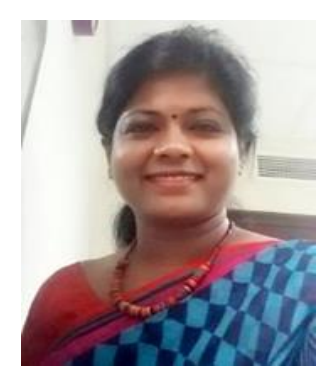

Dr R Swarnalatha did her BE in Instrumentation \& Control Engineering from Sathayabama Engineering College, Chennai and M.E in Instrumentation Engineering from Madras Institute of Technology, Anna University, Chennai. She received her PhD degree in Biomedical Instrumentation from Birla Institute of Technology and Science (BITS), Pilani, India. She has 20 years of teaching experience. She is working with BITS Pilani, Dubai Campus for past 14 years. She has guided many projects and taught various courses for undergraduate and postgraduate students. Her research interest includes biomedical instrumentation, process control \& Instrumentation, neural network \& fuzzy logic. 